\title{
Intervenciones cambiarias esterilizadas, teoría y evidencia: el caso de México
}

as possible. What the second duty is no one has as yet discovered.

Oscar Wilde (1894)

Santiago Capraro

Rodríguez

Universidad Nacional Autónoma de México, Facultad de Economía

santiago.capraro@gmail.com

\section{Ignacio Perrotini}

Hernández.

Universidad Nacional Autónoma de México, Facultad de Economía

iph@unam.mx.

\section{Resumen}

Los bancos centrales que aplican un marco de política monetaria de metas u objetivos de inflación niegan que las intervenciones en los mercados cambiarios sean un instrumento adicional de su praxis política para alcanzar los objetivos de política monetaria, so pretexto de que en un régimen de tipo de cambio flexible esas intervenciones no son efectivas. Este trabajo analiza, con base en evidencia empírica, el papel de las intervenciones cambiarias esterilizadas en el marco de la política monetaria del Banco de México. Nuestros resultados revelan que las intervenciones cambiarias esterilizadas sí han sido efectivas y que el tipo de cambio ha desempeñado un papel esencial en la estabilización de la inflación en México.

Palabras Clave: Tasa de Interés, Política Monetaria, Banco Central, Tipo de Cambio.

Clasificación JEL: E4, E5, F31. 


\title{
Sterilized exchange rate market interventions, theory and evidence: the case of Mexico
}

\begin{abstract}
The central Banks that apply a framework of monetary policy based on inflation targeting do not recognize that their foreign exchange market interventions represent a second monetary policy instrument used regularly with the aim of achieving monetary policy goals. The above on the grounds that under a flexible exchange rate regime foreign exchange market interventions are ineffective. The present paper looks into empirical evidence of the Banco de México's foreign exchange market interventions. It is shown, on the basis of econometric results, that those interventions are effective indeed; hence the nominal exchange rate appears to play an instrumental role in the stabilization strategy of the monetary authority.
\end{abstract}

KeyWords: Interest Rate, Monetary Policy, Central Banking, Foreign Exchange. JEL Classification: E4, E5, F31.

\section{Introducción}

Los bancos centrales que aplican un marco de política monetaria de metas u objetivos de inflación no admiten que las intervenciones en los mercados cambiarios sean un instrumento adicional de su praxis política; el Banco de México (BM) es uno de ellos.

La justificación de esta negativa estriba en el supuesto (inverosímil) de que cuando existe un régimen de tipo de cambio flexible o de libre flotación, las intervenciones cambiarias esterilizadas no son efectivas para controlar el tipo de cambio (cf. Svensson, 2001; Banco de México, Informe Anual, varios años; Sarno y Taylor, 2002; Hüfner, 2004). No obstante, los bancos centrales suelen realizar intervenciones cambiarias en forma regular. La cuestión es si estas intervenciones constituyen parte sustancial de su estrategia para conseguir los objetivos de política monetaria, es decir, la estabilidad de precios, en cuyo caso surge la interrogante acerca de cuál es el papel del tipo de cambio nominal en esa estrategia monetaria de los bancos centrales. Tal cuestión es uno de los temas menos estudiados en la literatura sobre modelos de metas de inflación y en el debate acerca de la efectividad de la conocida regla de Taylor (Taylor, 1999). 
En este trabajo se analiza, con base en la evidencia empírica disponible, el papel de las intervenciones cambiarias esterilizadas en el marco de la política monetaria del Banco de México. Los resultados revelan que las intervenciones esterilizadas sí han sido efectivas y que el tipo de cambio ha desempeñado un papel esencial en la estabilización de la inflación en México. Por lo tanto, las intervenciones en los mercados cambiarios constituyen un segundo instrumento regular e independiente de la política monetaria del Banco de México, lo cual explica el fenómeno conocido como "miedo a flotar" el tipo de cambio (Calvo y Reinhart, 2002), así como el hecho de que la tasa de interés no es el ancla (única) de la inflación en México.

El trabajo se divide en cinco secciones. La primera se refiere a esta introducción. En la segunda se discute el origen y los mecanismos de transmisión de las intervenciones esterilizadas; después, se presenta un análisis de las intervenciones esterilizadas en México durante 1996-2010; posteriormente, se realiza un estudio empírico de los efectos de las intervenciones sobre el tipo de cambio nominal (TCN) en México con base en un modelo EGARCH y en el efecto medio del tratamiento (EFT); finalmente, se ofrecen una serie de conclusiones.

\section{Intervenciones esterilizadas: origen y mecanismos de transmisión}

La crisis del sistema monetario y financiero internacional de Bretton Woods y la transición por parte de Estados Unidos y de las principales economías desarrolladas hacia el régimen de tipo de cambio flexible a partir del 15 de agosto de 1971 marcan el comienzo de la historia moderna de las intervenciones de los bancos centrales en los mercados cambiarios (Edison, 1993; Sarno y Taylor, 2001). En este sentido, la principal controversia sobre esas intervenciones gira en torno a su efectividad para afectar la trayectoria del tipo de cambio en la dirección deseada por la autoridad monetaria.

En las economías del Grupo de los diez (G10) ${ }^{1}$, han existido cuatro etapas importantes relacionadas con las intervenciones: 1) desde la crisis de Bretton Woods hasta fines de la década de los setenta, en la que la mayoría de los países realizaban intervenciones en forma individual o coordinada para apreciar o depreciar una mo-

\footnotetext{
${ }^{1}$ El G10 está conformado por Alemania, Bélgica, Canadá, Estados Unidos, Francia, Italia, Holanda, Japón, Reino Unido, Suecia y Suiza. Uno de los principales acuerdos firmados por el G10 fue el de Smithsonian en 1971, que dio por finalizado los acuerdos sobre tipos de cambio fijos establecido en Bretton Woods (véase los documentos del FMI en http://www.imf.org/external/np/exr/facts/groups.htm).
} 
neda; 2) desde fines de la década de los setenta hasta mediados de la década de los ochenta, cuando los bancos centrales disminuyeron su presencia en los mercados de divisas ${ }^{2}$; 3) desde 1985 hasta principios de la década de los noventa, cuando se realizaron los acuerdos del Hotel Plaza (1985) y de Louvre (1987); 4) desde mediados de la década de los noventa hasta el presente, el cual es un periodo dominado por intervenciones individuales ${ }^{3}$.

En las economías en desarrollo, las intervenciones surgen durante los años noventa debido a la creciente adopción - forzada en la mayoría de los casos a causa del colapso de los sistemas fijos- de regímenes de tipos de cambio flexibles.

Este artículo se centra en el caso de México, que adoptó un tipo de cambio flexible luego de la crisis de 1994-1995 como parte de una estrategia tendiente a practicar una política monetaria de metas de inflación.

Los banqueros centrales están convencidos de los efectos positivos de las intervenciones sobre el tipo de cambio (cf. Neely, 2005 y BIS, 2005). Existe consenso acerca de que las intervenciones son útiles para afectar el tipo de cambio y no incrementan su volatilidad. Los participantes del mercado, en cambio, tienen otra opinión: Cheung y Chinn (2001) encuestaron a corredores financieros en Estados Unidos y encontraron que el $61 \%$ de los encuestados sostiene que las intervenciones incrementan la volatilidad del tipo de cambio y no logran la meta deseada por la autoridad monetaria.

A pesar de que los bancos centrales hacen un amplio uso de las intervenciones como instrumento de política monetaria para controlar el tipo de cambio, no existe un consenso entre los economistas acerca de la efectividad de las mismas. El uso de las intervenciones ha generado un gran debate en el ámbito académico que rebasa al hecho de su efectividad o no, ya que si son efectivas, tampoco existe un acuerdo de cuál es la forma óptima de llevarlas a cabo, puesto que las intervenciones pueden ser secretas o públicas, o realizarse en el mercado spot o forward (Archer, 2005; Domínguez y Panthaki, 2007; Vitale, 2006).

\footnotetext{
${ }^{2}$ En 1982 se publicó el Jurgensen Report que estimaba que las intervenciones tenían un efecto leve en el muy corto plazo y que las intervenciones coordinadas eran más efectivas que las individuales (Sarno y Taylor, 2001).

${ }^{3}$ Con el fin de evitar la apreciación del yen, Japón realizó las mayores intervenciones en volumen para países desarrollados (Sarno y Taylor, 2001).
} 
El análisis de la efectividad de las intervenciones esterilizadas nos remite al balance de un banco central - simplificado - constituido por tres cuentas: los activos internacionales netos (AIN) y el crédito interno neto (CIN), compuesto por préstamos a los bancos comerciales, en el lado del activo, y, en el pasivo, la base monetaria (BM), que incluye el dinero en poder del público más los depósitos de la banca en sus cuentas corrientes en el banco central.

Las ecuaciones (1) y (2) muestran los movimientos en las variables relevantes, las cuales determinan que una intervención sea esterilizada o no. La expresión (1) define a las intervenciones esterilizadas; se observa que una variación en los $A I N_{t}$ está asociada a una variación igual del $C I N_{t}$, pero de signo contrario, determinando así que la base monetaria $\left(B M_{t}\right)$ no se modifique (esterilización total):

$$
\Delta A I N_{t}>/<0 ; \Delta C I N_{t}=-\Delta A I N_{t} ; \Delta B M_{t}=0,
$$

$$
\left.\left.\Delta A I N_{t}\right\rangle /<0 ; \Delta C I N_{t}=0 ; \Delta B M_{t}\right\rangle /<0 \text {. }
$$

La expresión (2) representa el caso de las intervenciones no esterilizadas; ante una variación de los $A I N_{t}$ el $C I N_{t}$ no varía en la misma proporción, por tanto la $B M_{t}$ varía.

Dada la definición de las intervenciones esterilizadas, la visión tradicional monetaria sostiene que no pueden ser efectivas. Como las intervenciones esterilizadas no afectan la base monetaria, éstas no causan una variación en el tipo de cambio. Dicha conclusión se alcanza si el tipo de cambio efectivamente se determina a través del enfoque monetario, el cual parte de la teoría de la paridad del poder de compra (PPC) y de la teoría cuantitativa del dinero; por tanto, si tenemos que:

$$
\begin{gathered}
P=e P^{*}, \\
P Y=M V, \\
P^{*} Y^{*}=M^{*} V^{*} .
\end{gathered}
$$


Donde $M$ es la base monetaria, $V$ es la velocidad de circulación del dinero, $P$ es el nivel de precios nacional, $e$ el tipo de cambio nominal y $Y$ es el nivel de producción local (las variables con asterisco corresponden a la economía extranjera). La ecuación (3) es la PPC y las expresiones (4) y (5) representan la condición de equilibrio en el mercado de dinero nacional y en el de la economía extranjera, respectivamente. Reemplazando (4) y (5) en (3) se obtiene la determinación del tipo de cambio según este enfoque:

$$
e=\frac{M}{M^{*}} \frac{V}{V^{*}} \frac{Y^{*}}{Y} .
$$

Por lo tanto, dado que una intervención esterilizada no modifica $M$ no puede afectar al tipo de cambio. Desde el punto de vista teórico existe aquí una incoherencia, esto es, argumentar en contra de las intervenciones cambiarias en el contexto de un marco de política monetaria de metas de inflación es paradójico, puesto que su rechazo recurre a la teoría cuantitativa del dinero, la cual descansa en el supuesto de exogeneidad de la moneda ajeno al modelo de metas de inflación basado en la regla de Taylor. Se trata pues de un non sequitur. ${ }^{4}$

Al principio de la literatura sobre intervenciones se plantearon dos mecanismos de transmisión para explicar la efectividad de las mismas, las cuales fueron denominados portafolio de equilibrio (Domínguez y Frenkel, 1993) y el canal de las señales (Edison, 1993). Sin embargo, estos mecanismos no lograron un consenso, por lo que se propusieron dos mecanismos adicionales: la existencia de inversionistas con racionalidad no tradicional (IRNT) o noise-traders (Harvey, 2008) y el canal de los flujos de pedidos o de la microestructura del mercado del tipo de cambio (Sarno y Taylor, 2001).

El canal del portafolio de equilibrio es similar al enfoque monetarista, pero modifica el supuesto de inversionistas neutrales al riesgo; por lo tanto, los activos nacionales y extranjeros no son sustitutos perfectos (Gandolfo, 2000). Según este enfoque, si un banco central realiza una intervención para apreciar su moneda y

\footnotetext{
${ }^{4}$ Gandolfo (2000, p. 227): "El enfoque monetario requiere que los activos nacionales y extranjeros sean sustitutos perfectos [...] este es un supuesto fuerte, en tanto implica que los inversionistas sean indiferentes a los tipos de activos que poseen en su portafolio (siempre que los activos nacionales y extranjeros tengan la misma tasa de rendimiento esperada expresada en una moneda común). Esto, a su vez, requiere que se cumpla la paridad descubierta de tasas de interés" [PDI; itálicas agregadas]. Por tanto, si la PDI no se cumple tampoco se sigue la conclusión de este enfoque.
} 
luego esteriliza esa operación, modificará la relación de equilibrio entre los activos externos e internos que existía antes de la intervención. En este caso, aumenta la proporción de activos extranjeros respecto a los nacionales. Por ello, los inversionistas venderán activos internacionales en su poder para retornar a la proporción anterior a la intervención; ello provocará, por un lado, que se incremente la tasa de interés sobre esos activos y, por otro, una depreciación de la moneda extranjera o una apreciación de la moneda local, lo cual es el objetivo del banco central. Si bien este mecanismo ofrece una explicación plausible de cómo las intervenciones afectan al tipo de cambio, el canal del portafolio de equilibrio ha tenido poco respaldo empírico (Edison, 1993). Un problema fundamental de esta explicación es que se basa en un cambio en la cantidad de bonos nacionales y extranjeros, pero el volumen de las intervenciones es nimio en relación con la cantidad de esos activos (Sarno y Taylor 2001).

El canal de las señales plantea que cuando un banco central interviene en el mercado de tipo de cambio pretende indicar cuál será su postura de política monetaria en el futuro; de esta forma afecta a las expectativas sobre el tipo de cambio futuro y, por lo tanto, sobre el tipo de cambio corriente. Este canal tiene falencias teóricas insalvables por dos motivos: primero, no explica por qué la mayoría de los bancos centrales mantiene en secreto su actividad en los mercados de divisas, por lo que cómo puede un banco central dar una señal a los mercados ocultando al mismo tiempo esa señal (Domínguez y Frankel, 1993); segundo, según este enfoque no hay necesidad de que las intervenciones sean esterilizadas, pero en la práctica los bancos centrales que intervienen tienden a esterilizar parcial o totalmente sus operaciones.

Los mecanismos restantes ponen mayor atención en las características particulares de los mercados financieros y en su incidencia en la determinación del tipo de cambio. Por ejemplo, un hecho estilizado de los mercados financieros es la existencia de IRNT, la particularidad de estos inversionistas es que su posición en el mercado (su demanda u oferta) de divisas está influida por sus creencias o sentimientos que pueden no ser consistentes con las variables relevantes del mercado, como puede ser el valor del tipo de cambio que predice la ecuación de la PDI $(\boldsymbol{e}=\boldsymbol{\alpha}(\boldsymbol{i}-\boldsymbol{i} \boldsymbol{*})$ ) (Hung, 1997; Harvey, 2008). Al existir este tipo de inversionistas, el valor del tipo de cambio puede desviarse de su valor consistente con la información del mercado por un periodo de tiempo prolongado; por lo tanto, si un banco central aplica una política de regla de Taylor, las intervenciones se pueden 
utilizar para evitar que la actividad de los IRNT ponga en riesgo el cumplimiento de la meta de inflación.

Sin embargo, el enfoque IRNT es más relevante cuando se considera la efectividad de las intervenciones. Como los IRNT actúan con base en la tendencia del mercado, el objetivo específico de las intervenciones esterilizadas es provocar un cambio en esa medida que revierta una devaluación o apreciación no deseada. Los inversionistas IRNT pueden apoyar un efecto en cascada iniciado por una intervención del banco central en el mercado cambiario, de suerte que una intervención esterilizada prolongue sus efectos más allá de lo planeado inicialmente por el propio banco central (Hüfner, 2004).

El canal de la microestructura se centra en la actividad de los intermediarios en el mercado de divisas y en la determinación diaria del tipo de cambio. Tres características distinguen a este enfoque: primero, supone que existen diferentes tipos de participantes en el mercado: intermediarios, INRT y participantes comerciales — relacionados con actividades de importación y exportación-; segundo, cada tipo de participante posee una cantidad diferente de información y además con la misma información pueden tomar posiciones diferentes en el mercado; y tercero, una de las variables clave es el flujo de pedidos en el mercado de cambios, variable que no está disponible para muchos países en desarrollo (Harvey, 2008). Con los nuevos elementos que plantea el canal de la microestructura algunos autores han reconsiderado los canales anteriores; sin embargo, destaca en particular una revalidación empírica del canal del portafolio (Lyons, 2001; Vitale, 2006). Asimismo, para que las intervenciones sean efectivas es importante, en primer lugar, el tipo de agente que realiza el pedido en nombre del banco central y, luego, la manera en cómo se dispersa la información (Lyons, 2001).

Finalmente, un quinto mecanismo es el canal de acumulación de reservas a través del cual un banco central puede controlar la evolución del tipo de cambio con las intervenciones esterilizadas utilizando los canales de señalización, el de los IRNT y el de microestructura. La acumulación de reservas indica que el banco central está dispuesto a intervenir en el mercado de cambios cuando sea necesario; este mecanismo ha sido tratado en la literatura sobre "miedo a flotar" (Calvo et al., 2002). Los mecanismos de IRNT y microestructura juegan aquí un papel en las intervenciones de venta de divisas. Por lo tanto, más que un mecanismo de transmisión, de lo que se trata es de un régimen de intervención para controlar el tipo de cambio. 
La incertidumbre sobre cuál de los canales es el que efectivamente opera cuando el banco central interviene, refleja dos aspectos importantes: primero, la falta de relevancia que se confiere en general en la literatura oficial y aún en la heterodoxa a las intervenciones cambiarias como un mecanismo para controlar el tipo de cambio, por lo que la teoría económica debería enfatizar más el papel de este importante instrumento de política monetaria (Domínguez y Frankel, 1993); segundo, la dificultad que plantean las intervenciones cambiarias a la investigación empírica (Neely, 2005).

\section{Intervenciones esterilizadas en México, 1996-2010}

Una de las consecuencias más relevantes de la crisis de balanza de pagos de 19941995 fue el abandono del régimen de tipo de cambio semifijo y la adopción a partir de 1995 de un régimen de flotación libre (cf. Programas de Política Monetaria de1995 a 2010).

El nuevo régimen está a tono con el trípode fundamental de la llamada regla de Taylor (1999), la cual afirma que la única política monetaria posible que puede implementar un país que abandonó un tipo de cambio fijo se basa en un tipo de cambio flexible, una regla de política monetaria y una meta de inflación.

Sin embargo, los bancos centrales suelen no practicar la política cambiaria (de libre flotación) que pregonan (Calvo y Reinhart, 2002). En teoría, en un régimen de flotación libre (RFL) las reservas internacionales del banco central tendrían que ser constantes y poco relevantes como variable de política monetaria, lo cual no ha ocurrido en los países desarrollados y en desarrollo que durante los últimos 20 años se autodenominan flotadores libres. México es un claro ejemplo: durante el periodo de flotación del peso las reservas internacionales del BM se han incrementado desde 6,313 millones de dólares (md) en 1996 a 120,621 md en 2010 (ver cuadro 1).

De la gráfica 1 se infiere que durante el periodo 1996-2010 el BM intervino de forma continua en el mercado de divisas, excepto entre junio de 2001 y mayo de 2003. El BM realizó intervenciones en el mercado de divisas mediante nueve mecanismos diferentes ${ }^{5}$ :

\footnotetext{
${ }^{5}$ Ver sección Operaciones del Banco de México en el mercado cambiario en www.banxico.org.mx.
} 
1. Subastas de opciones de venta de dólares entre agosto de 1996 y junio de 2001, así como desde febrero de 2010 a diciembre de 2010.

2. Subastas de dólares entre febrero de 1997 y junio de 2001.

3. Mecanismo para reducir el ritmo de acumulación de reservas internacionales entre mayo de 2003 y julio de 2008.

4. Subastas de dólares extraordinarias en octubre de 2008.

5. Subastas de dólares con precio mínimo durante octubre de 2008 a abril de 2010.

6. Subasta de dólares sin precio mínimo, vigente de marzo de 2009 a septiembre de 2009.

7. Subasta de créditos en dólares por disposición de línea swap con la FED.

8. Venta directa de dólares en el mercado spot en septiembre de 1998 y febrero de 2009.

9. Línea de créditos flexibles que negoció la Comisión de Cambios (CC) con el FMI por 48,000 md, vigente de 2009 a 2010; sin embargo, cabe indicar que para el periodo de este estudio no se utilizó.

Dado el sesgo antiinflacionario de la política monetaria del BM, la política cambiaria se debe analizar teniendo en cuenta que el único objetivo del BM (y la CC) ha sido controlar los efectos inflacionarios del TCN. Por tanto, la naturaleza de las intervenciones en México difiere de la de otros países en donde se pretende evitar apreciaciones nominales del TCN (Adler y Tovar, 2011). Durante la aplicación de metas de inflación en México se ha dejado apreciar el TCN cuando han entrado capitales, y se ha intervenido cuando el tipo de cambio se ha depreciado por la salida de capitales.

Con excepción de Mántey (2009), en los análisis de la política monetaria de México no se ha otorgado suficiente importancia a las intervenciones cambiarias como herramienta para controlar el tipo de cambio y, a través de éste, controlar la inflación. Domaç y Mendoza (2004) y Guimarães y Karacadag (2004) estudian las intervenciones cambiarias en México y concluyen que no son efectivas para controlar el tipo de cambio. Cabe señalar que Galindo y Ros (2008) analizan la política cambiaria del BM en forma distinta a la que proponemos en este trabajo. Ellos afirman que existe una política monetaria asimétrica de la tasa de interés con relación al tipo de cambio, esto es, la tasa de interés se incrementa cuando hay presiones devaluatorias, apreciando así el TCN, pero el BM no disminuye la tasa de interés cuando el TCN se aprecia. Perrotini (2012) distingue entre la política monetaria del $\mathrm{BM}$ respecto a los periodos con estabilidad y la política monetaria durante las 
crisis, y concluye que en los momentos de crisis la política monetaria se concentra en la tasa de interés. En el presente trabajo se propone que además de una política asimétrica existe también una política cambiaria basada en las intervenciones cambiarias que no reemplaza a la primera sino que la complementa; postulamos, además, que las intervenciones cambiarias son un segundo instrumento de política monetaria y el tipo de cambio un objetivo intermedio.

Existen diversas razones por las cuales un banco central realiza intervenciones en el mercado de divisas; destacamos dos que parecen las más relevantes. Primero, la hipótesis de paridad descubierta de tasas de interés (PDI) no se convalida; por lo tanto, si el banco central manipulara la tasa de interés para controlar el tipo de cambio no lograría su cometido, ya que los flujos de capital no responden solamente al diferencial de tasas de interés, sino que también responden a otras variables (por ejemplo, la prima de riesgo, el fenómeno conocido como flight to quality). Segundo, aun si la PDI se cumpliera un banco central puede preferir realizar intervenciones en el mercado de divisas y no provocar movimientos en la tasa de interés para contrarrestar movimientos no deseados en el TCN, ya que alterar la tasa de interés puede contravenir otros objetivos de política como alcanzar un nivel de producción de pleno empleo. En el caso de México, sostenemos que la primera razón para realizar intervenciones cambiarias es la más relevante. (cf. También Benavidez y Capistrán, 2009).

La hipótesis fundamental de nuestra investigación es que las intervenciones cambiarias son un instrumento independiente de la tasa de interés que sirve para controlar el tipo de cambio, evitando así depreciaciones no deseadas del TCN que ponen en riesgo la consecución del objetivo de inflación. El aspecto más controvertido de la afirmación anterior es determinar si las intervenciones de venta determinan que el tipo de cambio se aprecie, pues en la literatura sobre el tema la mayoría de los autores comparte la visión de Svensson (2001): “...no hay razones para que un banco central que aplique metas de inflación de forma responsable y transparente deba realizar intervenciones en el mercado de tipo de cambio" y sostienen que las intervenciones no son efectivas (Nelly 2005). En consecuencia, a continuación analizamos el efecto de las intervenciones de venta de dólares sobre el TCN en el periodo 1996-2010, para lo cual utilizamos dos metodologías: una de series de tiempo (GARCH) y otra de estudios de evento (EMT). 


\section{Un modelo GARCH para estimar el impacto de las intervenciones sobre el tipo de cambio}

La gráfica 1 muestra la evolución diaria de la variación del TCN en México en el periodo 1996-2010; de ésta se puede inferir que han existido periodos de tranquilidad y periodos de aumento en la volatilidad. También se identifican dos periodos en los que aumentó la volatilidad del tipo de cambio: (1) hacia finales de 1997 y durante 1998, así como (2) durante 2008 y 2009; en 2010 el tipo de cambio parece volver a su nivel de volatilidad normal. Las características que muestra la variación del tipo de cambio revelan que los modelos GARCH son una buena alternativa para investigar el impacto de las intervenciones en el tipo de cambio, ya que éste pareciera estar generado por un proceso de información heteroscedástico ${ }^{6}$.

El modelo GARCH estimado se conformó con las siguientes ecuaciones y variables:

$$
\begin{gathered}
s_{t}=\beta_{0}+\beta_{1} I V_{t-1}+\beta_{2} \text { difti }_{t}+\beta_{3} i p y c_{t}+\beta_{4} o_{t}+\varepsilon_{t} \\
\varepsilon_{t}=v_{t} \sqrt{h_{t}} \\
h_{t}=\psi_{0}+A R C H q+G A R C H p+\psi_{1} I V_{t-1}+\psi_{2} \text { difti }_{t}+\psi_{3} i p y c_{t}+\psi_{7} p \\
v_{t} \text { es un proceso ruido blanco con } \sigma^{2}=1
\end{gathered}
$$

Donde $s_{t}$ y $h_{t}$ son las variables endógenas del modelo y representan la variación diaria del tipo de cambio y la varianza condicional de $\varepsilon_{t}$, respectivamente. Las variables exógenas son: $I V_{t-1}$ que representa las intervenciones de venta de dólares mayores a 200 millones de dólares (md) por parte del BM rezagadas un periodo; difti $_{t}$ es la variación del diferencial de tasas de interés, entre la tasa local y la tasa internacional; $i p y c_{t}$ es la variación del Índice de Precios y Cotizaciones de la Bolsa Mexicana de Valores y $o_{t}$ es la variación del precio del petróleo tipo West Texas Intermediate (wti). Al incluir la variable difti $i_{t}$ en el modelo GARCH estimado se procedió también a comprobar la PDI. Finalmente, siguiendo a Bonser-Neal y Tanner (1996) y Frenkel et al. (2003), se incluyó en los modelos el Índice de Precios y

\footnotetext{
${ }^{6}$ Domaç y Mendoza (2004) y Guimarães y Karacadag (2004) también estudian el impacto de las intervenciones sobre el tipo de cambio en México a través de modelos GARCH, pero sólo analizan el periodo 1996-2001.
} 
Cotizaciones de la Bolsa Mexicana de Valores para controlar el impacto de eventos económicos o políticos en la volatilidad en todos los mercados financieros.

A priori se espera que $\beta_{2}$ no sea significativo, dado que el cumplimiento de la paridad descubierta de tasas de interés depende de supuestos no validados en la economía real. Dado que suponemos que las intervenciones del BM fueron efectivas en el periodo 1996-2010, se espera que el parámetro que acompaña a las intervenciones $\left(\beta_{1}\right)$ sea significativo. Independientemente del mecanismo a través del cual las intervenciones afectan al tipo de cambio, una intervención compra (venta) debería depreciar (apreciar) el tipo de cambio. Por tanto, esperamos que $\beta_{1}<0$.

El signo de $\beta_{4}$ se espera que sea negativo. De esta manera, un incremento (disminución) del precio del petróleo tiene el efecto de apreciar (depreciar) el tipo de cambio. Es importante destacar que la principal fuente de divisas del BM entre 1996-2009 fueron los ingresos por exportaciones de PEMEX (ver cuadro 1). Entonces, si aumenta el precio del petróleo, los participantes del mercado estiman que el $\mathrm{BM}$ tendrá disponible un mayor volumen de divisas internacionales para intervenir. De esta forma una baja del precio indica lo contrario. En conclusión, la evolución del precio del petróleo afecta al tipo de cambio a través de las expectativas de los agentes sobre la reacción futura del BM.

\section{Datos y resultados}

El periodo de estudio se extiende desde el 04/06/1996 hasta el 31/12/2010. Los datos de las series contienen 3670 observaciones. La información del tipo de cambio se obtuvo del BM, se utilizó el tipo de cambio FIX ${ }^{7}$, que es un precio del mercado mayorista donde ocurre la casi totalidad de las intervenciones.

\footnotetext{
${ }^{7}$ Según el BM: "El tipo de cambio FIX es determinado por el Banco de México los días hábiles bancarios con base en un promedio de las cotizaciones del mercado de cambios al mayoreo para operaciones liquidables el segundo día hábil bancario siguiente. Dichas cotizaciones se obtienen de plataformas de transacción cambiaria y otros medios electrónicos con representatividad en el mercado de cambios. El Banco de México da a conocer el FIX a partir de las 12:00 horas de todos los días hábiles bancarios."'( http://www.banxico.org.mx/indicadores/fix. html).
} 
El BM es uno de los pocos bancos centrales en el mundo 8 que publica regularmente información sobre su intervención en el mercado de divisas (ver cuadro 2). En todo el periodo el BM intervino en 1612 ocasiones, lo cual es significativo en comparación con otros bancos centrales ${ }^{9}$. Si consideramos subperiodos, basándonos en las políticas explícitas de acumulación/desacumulación de reservas y periodos de crisis, se pueden definir 4 regímenes de intervención (ver cuadro 2). El mayor número de intervenciones se dio durante el periodo de desacumulación de reservas; de un total de 1340 días hábiles el BM intervino en 1264, es decir, $94.33 \%$ del total de días. El periodo de menor intervención es el de acumulación de reservas con 143 episodios de intervención en 1242 días hábiles. Los datos muestran que las intervenciones de compra de dólares sólo sucedieron durante el subperiodo 19962001 y durante 2010; para el resto del periodo el BM intervino vendiendo reservas internacionales. Del universo de intervenciones considerado en el presente trabajo, analizaremos el efecto de las intervenciones de ventas mayores a 200 millones de dólares (md).

Utilizamos la tasa de interés interbancaria de equilibrio (TIIE) como tasa de referencia de México, la tasa de fondos federales a un día de la FED como referente de Estados Unidos y el wti como precio del petróleo. No fue posible conformar una serie del precio de la mezcla mexicana para todo el periodo. La información del precio del wti se obtuvo de la U.S. Energy Information Administration. Finalmente, la información sobre la Bolsa Mexicana de Valores procede del Instituto Nacional de Estadística Geografía e Informática (INEGI).

En el cuadro 3 se muestran los resultados de la estimación del modelo. El primer resultado que debe destacarse en la ecuación de la media condicional es que el coeficiente de las intervenciones de venta mayores a $200 \mathrm{md}(I V)$ es estadísticamente significativo y tiene el signo esperado. El valor del coeficiente es -0.002345 , lo que implica que una $I V$ provocó una apreciación diaria del TCN de $0.5 \%$ durante el periodo de estudio. Entre 1996 y 2010 hubo 19 episodios en que el BM realizó intervenciones por $400 \mathrm{md}$ que, con base en los resultados obtenidos, provocaron

\footnotetext{
${ }^{8}$ Por ejemplo, Japón apenas en 2003 publicó los datos referidos al periodo 1991-2003 y a partir de esa fecha publica los datos de intervenciones en forma regular (Fatum y Hutchinson, 2006; Sarno y Taylor, 2001).

9Japón -con el mayor volumen de intervenciones entre los países desarrollados-intervino entre finales de la década de 1990 y comienzos de la década de 2000 sólo en 159 días (Fatum, 2010).
} 
una apreciación diaria del 1\%. Este monto no parece significativo, pero lo importante es que revierte la tendencia del TCN a la depreciación. Dos ejemplos reflejan el resultado obtenido en los modelos GARCH. Primero, el 10 de octubre de 2008 el BM intervino en el mercado a través de subastas por $6,400 \mathrm{md}$, al día siguiente el TCN se apreció 6\%, el modelo prevé una apreciación mayor del 15\%. Segundo, entre el 3 y el 6 de febrero de 2009 el BM intervino todos los días con ventas de 365 md en promedio, lo que produjo una apreciación diaria promedio de $0.7 \%$; la simulación del modelo anticipa una apreciación promedio de $0.85 \%$.

Un aspecto que no coincide con otros estudios (cf. Domaç y Mendoza, 2004 y Guimarães y Karacadag, 2004) es que el costo ${ }^{10}$ de las intervenciones en términos de incrementos en la volatilidad del TCN es nulo. De hecho, como se observa en el cuadro 3, las intervenciones mayores a 200 md disminuyen la volatilidad en un factor igual a 0.0005. La diferencia en los resultados obtenidos seguramente radica en que los estudios mencionados sólo analizan el periodo de acumulación de reservas 1996-2001 y, por tanto, no tuvieron en cuenta las $I V$ realizadas en 2008 y 2009.

Respecto a la variable difti el signo esperado es el que en general se postula en la teoría neoclásica, es decir, se satisface la hipótesis PDI. Sin embargo, el coeficiente no es estadísticamente significativo al $95 \%$. Claramente, este resultado requiere mayor investigación. El resto de las variables consideradas resultaron estadísticamente distintas de cero y del signo esperado. Finalmente, es necesario mencionar que a diferencia de otros trabajos (Nelly 2005), el modelo con mejor ajuste fue un EGARCH $(2,1)$ con un coeficiente de asimetría, lo que revela que las devaluaciones tienen un efecto más pronunciado sobre la volatilidad del TCN que las apreciaciones. Asimismo, el modelo no presenta heterocedasticidad en los errores ${ }^{11}$.

\section{El efecto medio de las intervenciones sobre el tipo de cambio}

En la presente sección se estudia la relación entre las intervenciones y el tipo de cambio mediante una metodología de estudio de eventos llamada efecto medio del tratamiento (EMT), estimado con agrupamientos a través de la propensity score (PS). Esta metodología se ha empleado para analizar el efecto de las intervenciones

\footnotetext{
${ }^{10}$ Para un análisis más amplio del costo de las intervenciones en México ver Mántey (2009).

${ }^{11}$ Las pruebas de evaluación de los errores del modelo dan cuenta de que no existe persistencia de heteroscedástidad. Las pruebas están disponibles para quien las solicite a los autores.
} 
sobre el tipo de cambio en Japón por Fatum y Hutchinson (2006) ${ }^{12}$. La búsqueda y uso de metodologías alternativas se debe a la ausencia de resultados satisfactorios con base en modelos de series de tiempo tradicionales que probaran la existencia de relación entre las intervenciones y el tipo de cambio (Nelly 2005) ${ }^{13}$.

Dos son los aspectos que nos motivan a estudiar el efecto de las intervenciones sobre el TCN mediante el EMT: primero, ningún modelo econométrico se ajusta perfectamente a las características de las variables consideradas en la investigación; segundo, un argumento contra-fáctico: partiendo de los tipos de cambio observados es imposible saber cuáles habrían sido las fluctuaciones efectivas del tipo de cambio en el hipotético caso de que las intervenciones cambiarias no hubieran tenido lugar (Calvo y Reinhart, 2002). El EMT es un método alternativo para conformar un conjunto eficiente de observaciones contrafactuales y así poder evaluar lo que hubiese ocurrido si el BM no hubiera intervenido.

Una de las principales características de los estudios de evento es que no se basan en relaciones estructurales. Como se ha planteado más arriba, no tienen como fundamento una forma funcional para el tipo de cambio, lo que es clave para los modelos de series de tiempo. El EMT estimado a través de la $P S$ analiza la relación entre las variables de forma contrafactual, comparando la evolución del tipo de cambio los días en que hubo intervenciones con aquellos días sin intervenciones, pero que son similares en términos de un conjunto de variables. El método tiene tres ventajas. Primero, un elemento instrumental del método consiste en el cálculo de la $P S$, que es la probabilidad condicionada sobre las variables exógenas de la ocurrencia de una intervención; por lo tanto, requiere estimar una función de reacción del $\mathrm{BM}^{14}$, obteniendo así un modelo que muestra cómo varía la probabilidad de que el BM intervenga cuando varían las variables exógenas consideradas.

\footnotetext{
${ }^{12}$ Las primeras aplicaciones del EMT en economía se hicieron en el campo del análisis financiero. Otros autores lo han aplicado en diversos temas, como la liberalización de la cuenta capital y la probabilidad de ocurrencia de una crisis monetaria (Glick et al. 2004). Lin y Ye (2011) utilizan la metodología del EMT para evaluar si los regímenes de metas de inflación fueron importantes para disminuir la inflación en países industriales y en desarrollo.

${ }^{13}$ El EMT pertenece a un campo de conocimiento estadístico/econométrico denominado estudios de evento; la bibliografía que aborda el tema de las intervenciones utilizando elementos de ese campo es amplia (cf. Ito y Yabu, 2004; Humpage et al., 2005; Kim, 2003).

${ }^{14} \mathrm{Si}$ bien se pueden hacer modelos que impliquen múltiples tratamientos, para el cálculo del EMT se recomienda estimar modelos separados para los distintos tratamientos (Caliendo, 2005).
} 
Segundo, un aspecto interesante del método es que a través de agrupar días con y sin intervención, por su cercanía en relación al valor de la PS, la diferencia que exista entre esas observaciones sólo será atribuible a las intervenciones. Es relevante analizar brevemente esta segunda característica para comprender el método utilizado $^{15}$. Supongamos que la variación del tipo de cambio se puede definir a través de la siguiente forma funcional:

$$
s_{t}=F\left(X_{t}\right)+w_{t} g\left(I_{t}\right) .
$$

Donde $s_{t}$ es la variación del tipo de cambio en el momento $t ; F\left(X_{t}\right)$ es una función vectorial de variables exógenas; $w_{t}$ es un indicador de la existencia de intervención en el momento $t$, el cual toma el valor 1 si hubo intervención y 0 en caso contrario; finalmente $g\left(I_{t}\right)$ es una función de las intervenciones que presenta la siguiente forma:

$$
g\left(I_{t}\right)=\beta I_{t}
$$

Si se supone que tenemos dos momentos $t=(j, i)$, con $w_{j}=1$ y $w_{i}=0$. Por tanto tendremos:

$$
\begin{aligned}
& s_{j}=F\left(X_{j}\right)+g\left(I_{j}\right), \\
& s_{i}=F\left(X_{i}\right) .
\end{aligned}
$$

Si ahora hacemos la diferencia entre los dos momentos, obtendremos:

$$
s_{j}-s_{i}=F\left(X_{j}\right)-F\left(X_{i}\right)+g\left(I_{j}\right) .
$$

En la ecuación (15) se puede apreciar que la diferencia entre las variaciones del tipo de cambio se debe a las diferencias en las funciones $F\left(X_{t}\right)$ y al efecto de las intervenciones sobre la variación del tipo de cambio. Lo que hace el método es agrupar de tal forma que:

Por tanto:

$$
\begin{gathered}
F\left(X_{j}\right)-F\left(X_{i}\right)=0 . \\
s_{j}-s_{i}=g\left(I_{j}\right)
\end{gathered}
$$

\footnotetext{
${ }^{15}$ El desarrollo teórico del EMT se puede encontrar en Rosenbaum y Rubin (1983), Becker e Ichino (2002), Imbens (2004), Wooldridge (2004) y Caliendo (2005).
} 
De esta manera, la diferencia entre las observaciones sólo depende de las intervenciones. Por último, la ecuación (17) evidencia que el método no requiere suponer que no existe una forma funcional para el tipo de cambio; de hecho se supone que existe, es $F\left(X_{t}\right)$, pero se la elimina en el proceso de calcular el EMT, que en el ejemplo es $g\left(I_{t}\right)$.

En el contexto de las intervenciones la variable sobre la que se realiza el tratamiento es la variación del tipo de cambio; el tratamiento serán las intervenciones y las variables exógenas que se consideraron para estimar la PS, es decir, la función de reacción del $\mathrm{BM}$, son la variación del tipo de cambio rezagada un periodo y una variable que indica cómo reacciona el BM ante desvíos del tipo de cambio respecto a su media anual.

En todos los casos se calcularon modelos logit para estimar la probabilidad de que en el momento $t$ ocurriera una intervención. Las intervenciones, al ser ellas el tratamiento, se consideraron con la siguiente estructura:

$$
I_{t}\left\{\begin{array}{c}
=1, \text { si hubo intervención } \\
=0, \text { si no hubo intervención }
\end{array}\right\}
$$

El modelo logit presenta la siguiente estructura:

$$
\mathrm{I}_{\mathrm{Jt}}=\beta_{0}+\beta_{1} \mathrm{~s}_{\mathrm{t}}+\beta_{2} \delta_{t}+\varepsilon_{t}
$$

Donde $\mathbf{s}_{\mathbf{t}-1}$ es la variación del tipo de cambio en el periodo anterior y $\delta_{t}$ es el desvío del tipo de cambio respecto a su media anual ${ }^{16}$ a partir de la estimación de la probabilidad de intervenir en el momento $t$, estimada mediante la ecuación (19). Se calculó el efecto medio del tratamiento sobre los días con tratamiento (EMTT) a través del algoritmo del vecino cercano (VC), donde el momento $t$ es un día con intervención. Debido a la forma funcional de (19), el resultado del efecto de las intervenciones sobre el TCN en el periodo $t$ puede estar afectado por un problema de simultaneidad entre las intervenciones y el TCN. Por tanto, se procedió a calcular el EMTT de las intervenciones sobre el TCN durante los cuatro días posteriores a una intervención.

\footnotetext{
${ }^{16}$ Ambas variables pasan las pruebas de raíz unitaria, por lo que pueden considerarse estacionarias. Las pruebas están disponibles a petición expresa a los autores.
} 


\section{Datos, software utilizado y resultados}

Los datos de las variables de intervención y variación del tipo de cambio, así como el periodo de estudio son los que se emplearon para estimar el modelo GARCH. Una nueva variable que trata de medir la existencia de una política de TCN objetivo por parte del BM, la cual se construyó con la siguiente estructura:

$$
\delta_{t}=\mathrm{e}_{\mathrm{t}}-\mathrm{E}_{\mathrm{t}}
$$

Donde $\mathrm{e}_{\mathrm{t}}$ es el logaritmo natural del tipo de cambio en el periodo $t$ y $\mathrm{E}_{\mathrm{t}}$ es el logaritmo natural del promedio anual del tipo de cambio. Los programas utilizados fueron: pscore de Becker e Ichino (2002) para analizar si la PS está balanceada; para el cálculo del EMTT se utilizó psmatch2 de Leuven y Sianesi (2003).

Para calcular la probabilidad condicional de que el BM intervenga en un día específico se estimó un modelo logit basado en la ecuación (19). Los resultados se presentan en el cuadro 4. Es interesante destacar que las dos variables consideradas resultaron altamente significativas a un nivel de 99\%. Dado que los coeficientes son complejos de interpretar, se calculó el incremento porcentual en la probabilidad de que el BM intervenga cuando las variables independientes se alteran en un desvío estándar. En el caso de la variación del tipo de cambio se producirá un incremento de $167.3 \%$ en la probabilidad de que el BM realice una intervención. Asimismo, un aumento en un desvío estándar (o 3.39 veces respecto a su media) de la probabilidad de que el BM intervenga se incrementa $153.9 \%$. Finalmente, en la gráfica 2 se muestra cómo al incrementarse la variación del tipo de cambio aumenta la probabilidad de que el BM realice una intervención.

En la literatura del EMT se utilizan tres indicadores: el EMT, el EMT sobre las observaciones tratadas (EMTT) y el EMT sobre las observaciones no tratadas (EMTNT). El más interesante es el EMTT en tanto que permite observar si el objetivo que se pretende con el tratamiento se alcanza o no. En el cuadro 5 se muestran los resultados del EMTT calculados mediante el algoritmo VC. Como se estableció más arriba, el EMTT de las intervenciones sobre el TCN en el día $t$ puede estar contaminado por un problema de simultaneidad entre las variables, por eso nos concentraremos en lo que sucedió en el día posterior a la intervención. En el momento $t+l$ la variación diaria del TCN cuando hubo intervenciones fue 
en promedio $0.1 \%$, lo que implica que el BDM intervino en días con alta volatilidad, ya que la media de la serie es $0.013 \%$. Asimismo, los días sin intervenciones presentan una media de $0.013 \%$, lo cual revela que cuando hubo intervenciones la volatilidad del tipo de cambio fue 10 veces mayor que un día sin intervenciones. La diferencia entre los días sin intervenciones es positiva, es decir, producen una depreciación del tipo de cambio, efecto contrario al pretendido por el BM. Sin embargo, al agrupar los días con intervención con los días sin intervención, pero con una probabilidad de que el BM intervenga similar a los días con intervención, los resultados son diametralmente opuestos.

Los días en que no hubo intervenciones, pero que tenían una probabilidad de intervención similar a los días con intervenciones, el tipo de cambio se depreció en promedio 2.02\%, lo que implica que el EMTT es igual a una apreciación de 1.916\%; es decir, las intervenciones no evitan que el tipo de cambio se deprecie, sino que evitan depreciaciones mayores a las que habrían sucedido si el BM no hubiese intervenido. Al segundo día de la intervención el EMTT sigue siendo negativo, igual a $-1.473 \%$, pero cabe mencionar que la intervención logra apreciar el TCN en promedio en $0.52 \%$. En ambos casos el EMTT es estadísticamente significativo, al 99\% el primer día y al 90\% el segundo día después de la intervención.

En los momentos $t+3$ y $t+4$ el EMTT no es significativo, pero es de signo positivo; es decir, que el tipo de cambio se depreció más cuando hubo intervenciones que cuando no las hubo. Finalmente, en el momento $t+5$ el resultado es significativo y de signo positivo. Asimismo, el EMTT acumulado de los 4 días posteriores a una intervención es una apreciación del TCN igual a $0.84 \%$ respecto a un escenario sin intervenciones. Además, si tenemos en cuenta los EMTT estadísticamente significativos el efecto se duplica hasta alcanzar $1.82 \%$.

A modo de conclusión, respecto a la efectividad de las intervenciones, se debe destacar que las dos metodologías utilizadas EGARCH y EMTT son coincidentes en revelar la efectividad de las intervenciones mayores a 200 md para modificar el tipo de cambio en la dirección deseada por el BM. El modus operandi que se desprende de las acciones del BM (y de la CC) es el siguiente: i) acumular la mayor cantidad de reservas posibles en los momentos de tranquilidad del mercado (es decir, aquellos que corresponden con momentos de apreciación y poca volatilidad), principalmente mediante la compra de dólares que hace el BM a PEMEX (y en menor medida a través de la venta de opciones de compra de dólares que hace el BM 
a los bancos comerciales ${ }^{17}$ ) y ii) intervenir en el mercado cuando el tipo de cambio presenta una tendencia a la devaluación para romper esa tendencia y así evitar que a través del coeficiente de traspaso de las fluctuaciones del tipo de cambio al nivel de precios se incremente la inflación. A continuación es necesario establecer el grado de esterilización de las intervenciones realizadas por el BM.

\section{Intervenciones esterilizadas: la relación entre los AIN y el CIN del BM}

En la presente sección se desarrolla un modelo econométrico para estudiar la relación entre los activos internacionales netos (AIN) y el crédito interno neto (CIN) del BM durante el periodo enero de 1996 a diciembre de 2010 con el objetivo de establecer la magnitud de la esterilización en la política monetaria del BM.

Hemos afirmado que las intervenciones cambiarias son un segundo instrumento de política monetaria independiente de la tasa de interés si y sólo si son esterilizadas, ya que de esta manera no se pone presión sobre el mercado de dinero y, por tanto, sobre la tasa de interés.

La base monetaria se define como la suma de los AIN y el CIN del BM. Por tanto, las intervenciones esterilizadas implican que la bolsa monetaria no varíe ante una alteración en los AIN. Es decir, ante alteraciones de los AIN, el CIN debe variar en la misma magnitud, pero en sentido contrario. La gráfica 3 muestra que la política del BM está enfocada a esterilizar casi la totalidad de la variación en los AIN con el objetivo de mantener sin cambios la base monetaria. En esta gráfica también se aprecia que hubo un crecimiento de dicha base, por lo que la esterilización no fue total. Para establecer la magnitud de la esterilización Bofinger y Wollmershäuser (2001) utilizan mínimos cuadrados ordinarios (MCO) en virtud de que las variables en diferencia son estacionarias. Este método también ha sido aplicado por Hüfner (2004). En Bofinger y Wollmershäuser (2001) se propone el siguiente modelo para estudiar el grado de utilización de las intervenciones esterilizadas por parte de los bancos centrales:

$$
\Delta C I N_{t}=\beta_{0}+\beta_{1} \Delta A I N_{t}+\beta_{2} \Delta C I N_{t-1}+\varepsilon_{t} ; \text { con } \varepsilon_{t}=\text { los errores del modelo }
$$

\footnotetext{
${ }^{17}$ En pocas oportunidades, el BM reconoce explícitamente que la principal fuente de sus reservas es la paraestatal PEMEX; sin embargo, en el comunicado de prensa del día 22/02/2010 de la CC se deja establecido que "...la fuente más importante de acumulación de reservas proviene de las compras de dólares que el Banco de México hace directamente a Petróleos Mexicanos..."
} 
Para el caso en que la esterilización es total se espera que $\beta_{0}=0$ y $\beta_{1}=-1$. Con base en los datos de México, se estima que $\beta_{1}$ sea negativo y cercano a menos uno, pero no igual a menos uno. Sin embargo, aquí utilizaremos un modelo vectorial de corrección de errores (VEC). Una diferencia importante entre el análisis de Bofinger y Wollmershäuser (2001) y la estrategia que seguimos en este artículo es que se investigó la posibilidad de la existencia de una relación de cointegración entre las variables, en tanto que las mismas en niveles tendrían que ser I(1). Asimismo, si estuvieran cointegradas la estimación con base en el modelo de la ecuación (21) representaría un problema de especificación (Enders, 2004).

Es importante establecer que el modelo no analiza la política de esterilización relacionada con las intervenciones tendientes a modificar el tipo de cambio, sino que analiza la política global de esterilización del BM. Por tanto, la relaciones encontradas en el modelo (21) indican qué acción se espera del BM cuando los AIN varían independientemente de que esa variación se origine por un aumento de la liquidación de exportaciones de PEMEX, una demanda de divisas extranjeras del gobierno federal, una intervención en el mercado de divisas o una variación en las reservas por otros motivos diferentes (ganancias de las reservas invertidas, variación en la valuación de las mismas, etc. ${ }^{18}$.

\section{Datos y resultados}

La información acerca de los datos mensuales nominales de los AIN, el CIN y la base monetaria se obtuvo del BM. A diferencia de los modelos para analizar la efectividad de las intervenciones, en los cuales se tuvo en cuenta el periodo a partir de julio/agosto de 1996, en los modelos subsiguientes se consideró el periodo enero de 1996 a diciembre de 2009. La diferencia se explica porque no se está analizando la política monetaria del BM en relación con las intervenciones cambiarias exclusivamente, sino que se estudia su política global de esterilización. Por tanto, resulta relevante obtener siete observaciones más al ampliar el periodo de estudio.

\footnotetext{
${ }^{18}$ Dado que la información está disponible se procedió a calcular un modelo con la siguiente característica:

$\Delta C I N_{t}=\beta_{0}+\beta_{1} \Delta I_{t}+\beta_{2} \Delta C I N_{t-1}+\varepsilon_{t}$; con $\varepsilon_{t}=$ los errores del modelo, donde $\Delta I_{t}$ representa la variación de las reservas por una intervención en el mercado de divisas. Sin embargo, como el modelo no resultó robusto, no se informan los resultados obtenidos.
} 
Las variables pasan todas las pruebas de raíz unitaria con un grado de significancia del $1 \%$. Por tanto, se pueden considerar I(1). Para construir el modelo de corrección de errores se procedió a realizar pruebas de cointegración pertinentes de las cuales resulta que existe un vector de cointegración. Las pruebas de cointegración más robustas son aquellas que suponen la existencia de un intercepto en el vector de cointegración. Así, tanto la prueba de la traza como la del vector máximo son coincidentes ${ }^{19}$.

En el cuadro 6 se muestran los resultados de la estimación del vector de cointegración normalizado para la variable CIN. El parámetro de la variable AIN presenta el signo esperado y es estadísticamente significativo al 99\%. El valor estimado es -0.72 , es decir, una disminución de los AIN en 100 millones de pesos producirá un aumento del CIN en $72 \mathrm{mp}$, lo que se ajusta a los datos; en tanto la base monetaria en el periodo estudiado aumentó por un creciente proceso de monetización de la economía (ver informe anual del BM de 2009). Por tanto, una estimación más cercana a menos uno sería engañosa y no se ajustaría a los hechos estilizados de la evolución de las variables.

\section{Conclusiones}

Hemos analizado, con base en la evidencia empírica disponible, el papel de las intervenciones cambiarias esterilizadas en el marco de la política monetaria del Banco de México. Los resultados de nuestra pesquisa estadística (modelos EGARCH y del efecto medio del tratamiento, EFT) nos permiten concluir que las intervenciones esterilizadas sí han sido efectivas, que la consecución del objetivo de inflación y, por lo tanto, el "éxito" del Banco de México con relación a la estabilización de los precios, dependen crucialmente del control del tipo de cambio nominal a través de estas intervenciones en los mercados cambiarios. En consecuencia, a contrapelo de la hipótesis convencional (Svensson, 2010; Woodford 2003), éstas constituyen un segundo instrumento regular e independiente de la política monetaria del Banco de México, lo cual explica tanto el fenómeno conocido como "miedo a flotar" el tipo de cambio (Calvo y Reinhart, 2002) cuanto el hecho de que la política monetaria (esto es, la tasa de interés) no es el ancla (única) de la inflación en México.

\footnotetext{
${ }^{19}$ Las pruebas de raíz unitaria, rezagos óptimos, cointegración y el modelo VEC completo estimado están disponibles a solicitud a los autores.
} 
De lo aquí expuesto, se infieren diversas líneas de investigación para futuros trabajos, a saber: i) las implicaciones de nuestros resultados para la transparencia y responsabilidad de la política monetaria (la efectividad de las políticas de intervención requiere un cierto grado de opacidad, en particular con relación al objetivo intermedio, por ejemplo, el tipo de cambio); ii) el análisis de la autonomía del Banco de México (autonomía respecto de qué agentes ¿el gobierno o los mercados financieros;?) y iii) el análisis de los efectos de las intervenciones tanto en el tipo de cambio nominal como en la macro economía mexicana.

\section{Cuadro 1}

Evolución del stock y los flujos de los activos internacionales netos $^{1}$ del BM (en millones de dólares)

\begin{tabular}{ccccccc}
\hline & $\begin{array}{c}\text { Saldos de } \\
\text { Activos } \\
\text { Internacionales } \\
\text { Netos }\end{array}$ & $\begin{array}{c}\text { Flujos Netos } \\
\text { de Activos } \\
\text { Internacionales } \\
\text { Netos }\end{array}$ & $\begin{array}{c}\text { Flujos } \\
\text { Netos de } \\
\text { PEMEX }\end{array}$ & $\begin{array}{c}\text { Flujos } \\
\text { Netos de } \\
\text { Gobierno } \\
\text { Federal }\end{array}$ & $\begin{array}{c}\text { Operaciones } \\
\text { de Mercado }\end{array}$ & $\begin{array}{c}\text { Otros } \\
\text { Flujos } \\
\text { Netos }\end{array}$ \\
\hline 1996 & 6,313 & 6,348 & 9,047 & $-2,707$ & 864 & -858 \\
1997 & 19,824 & 13,511 & 8,470 & 859 & 3,821 & 361 \\
1998 & 23,480 & 3,656 & 5,430 & $-3,307$ & 310 & 1,224 \\
1999 & 27,380 & 3,900 & 7,426 & $-6,506$ & 1,820 & 1,160 \\
2000 & 35,629 & 8,249 & 11,172 & $-6,802$ & 1,794 & 2,085 \\
2001 & 44,857 & 9,228 & 8,905 & $-2,401$ & 1,363 & 1,360 \\
2002 & 50,722 & 5,865 & 10,019 & $-6,212$ & - & 2,059 \\
2003 & 59,059 & 8,337 & 15,380 & $-5,823$ & $-3,218$ & 1,998 \\
2004 & 64,233 & 5,174 & 13,832 & $-3,240$ & $-6,712$ & 1,295 \\
2005 & 74,115 & 9,882 & 20,378 & $-7,290$ & $-4,402$ & 1,196 \\
2006 & 76,304 & 2,189 & 26,698 & $-20,192$ & $-8,014$ & 3,697 \\
2007 & 87,235 & 10,931 & 12,900 & $-3,648$ & $-4,240$ & 5,920 \\
2008 & 95,232 & 7,997 & 22,754 & $-4,772$ & $-18,674$ & 8,690 \\
2009 & 99,870 & 4,638 & 11,529 & 5,903 & $-16,246$ & 3,453 \\
2010 & 120,621 & 20,751 & 16,037 & 274 & 4,466 & -26 \\
\hline
\end{tabular}

1: Según el BM los activos internacionales netos se definen como la reserva internacional bruta más otras inversiones en moneda extranjera que corresponden a los créditos convenio que otorgue el BM a otros bancos centrales a plazo de más de seis meses, menos los adeudos del $\mathrm{BM}$ con el FMI y con bancos centrales derivados de créditos convenio recibidos con plazo a vencimiento de menos de seis meses.

Fuente: Elaboración propia con base en datos del BM. 


\section{Cuadro 2}

Características generales de las intervenciones realizadas por el Banco de México durante 1996-2010

\begin{tabular}{|c|c|c|c|c|c|}
\hline & \multicolumn{5}{|c|}{ Periodo } \\
\hline & $\begin{array}{l}\text { Todo el } \\
\text { Periodo }\end{array}$ & $\begin{array}{l}\text { Acumulación } \\
\text { de Reservas }\end{array}$ & $\begin{array}{c}\text { Desacumulación } \\
\text { de Reservas }\end{array}$ & Crisis & $\begin{array}{c}\text { Acumulación de } \\
\text { Reservas }\end{array}$ \\
\hline & $\begin{array}{l}1996 *- \\
2010\end{array}$ & $\begin{array}{l}\text { Julio/1996- } \\
\text { Junio/2001 }\end{array}$ & $\begin{array}{l}\text { Abril/2003- } \\
\text { Julio/2008 }\end{array}$ & $\begin{array}{c}\text { Octubre/2008- } \\
\text { Diciembre/2009 }\end{array}$ & $\begin{array}{c}\text { Enero/2010- } \\
\text { Diciembre/2010 }\end{array}$ \\
\hline Días con actividad & 3,670 & 1,242 & 1,340 & 304 & 252 \\
\hline $\begin{array}{l}\text { Días sin } \\
\text { intervenciones }\end{array}$ & 2,058 & 1,096 & 76 & 131 & 29 \\
\hline $\begin{array}{l}\text { Días con } \\
\text { intervenciones }\end{array}$ & 1,612 & 146 & 1,264 & 173 & 223 \\
\hline $\begin{array}{l}\text { Probabilidad de } \\
\text { Intervenir(x100) }\end{array}$ & 43.92 & 11.76 & 94.33 & 72.38 & 11.51 \\
\hline $\begin{array}{l}\text { Probabilidad de no } \\
\text { Intervenir (x100) }\end{array}$ & 56.08 & 88.24 & 5.67 & 27.62 & 88.49 \\
\hline $\begin{array}{l}\text { Intervenciones de } \\
\text { Compra }\end{array}$ & 161 & 132 & 0 & 0 & 29 \\
\hline $\begin{array}{l}\text { Intervenciones de } \\
\text { Ventas }\end{array}$ & 1,451 & 14 & 1,264 & 173 & 0 \\
\hline $\begin{array}{l}\text { Probabilidad de } \\
\text { IC (x100) }\end{array}$ & 9.99 & 90.41 & 0 & 0 & 11.51 \\
\hline $\begin{array}{l}\text { Probabilidad de } \\
\text { IV }(x 100)\end{array}$ & 90.09 & 9.59 & 100 & 100 & 0 \\
\hline \multicolumn{6}{|c|}{ Frecuencia de las intervenciones por su monto (millones de dólares) } \\
\hline Menos de 20 & 477 & 31 & 442 & 1 & 3 \\
\hline Entre 20.1 y 40 & 647 & 9 & 634 & - & 4 \\
\hline Entre 40.1 y 60 & 284 & 17 & 188 & 79 & 0 \\
\hline Entre 60.1 y 100 & 36 & 27 & - & 1 & 8 \\
\hline Entre 100.1 y 200 & 101 & 35 & - & 59 & 7 \\
\hline Entre 200.1 y 300 & 33 & 26 & - & 5 & 2 \\
\hline Entre 300.1 y 400 & 13 & - & - & 10 & 3 \\
\hline Entre 400.1 y 500 & 14 & 1 & - & 11 & 2 \\
\hline Entre 500.1 y 750 & 1 & - & - & 1 & - \\
\hline Entre 750.1 y 1000 & 1 & - & - & 1 & - \\
\hline Entre 1000.1 y 1500 & 1 & - & - & 1 & - \\
\hline Entre 1500.1 y 2000 & 2 & - & - & 2 & - \\
\hline Entre 2000.1 y 3000 & - & - & - & - & - \\
\hline Entre 3000.1 y 4000 & 1 & - & - & 1 & - \\
\hline Más de 4000.1 & 1 & - & - & 1 & - \\
\hline
\end{tabular}

Fuente: Elaboración propia con base en información del BM. * El periodo de estudio se extiende desde 04/06/1996 al 31/12/2010. 


\section{Cuadro 3}

\section{Estimación del modelo GARCH}

\begin{tabular}{|c|c|c|c|}
\hline \multicolumn{4}{|c|}{ Ecuación de la media } \\
\hline $\mathbf{I V}_{(\mathbf{t}-\mathbf{1})}$ & difti $_{(\mathbf{t}-\mathbf{1})}$ & ipyc $_{\mathbf{t}}$ & $\mathbf{o}_{\mathbf{t}}$ \\
\hline$-.002345^{*}$ & $0.003083^{*} * *$ & -0.078708 & $0.010277^{*}$ \\
\hline$(0.00023)$ & $(0.00017)$ & $(0.003486)$ & $(0.002421)$ \\
\hline
\end{tabular}

\begin{tabular}{|c|c|c|c|c|c|c|c|}
\hline \multicolumn{7}{|c|}{ Ecuación de la varianza condicional } \\
\hline Constante & ARCH $_{\mathrm{t}-1}$ & $\mathbf{A R C H}_{\mathrm{t}-2}$ & $\mathbf{F A}$ & $\mathrm{GARCH}_{\mathrm{t}-1}$ & $\mathrm{IV}_{\mathrm{t}-1}$ & difti $_{\mathrm{t}-1}$ & $\mathrm{O}_{\mathrm{t}}$ \\
\hline$-0.308102 *$ & $0.322658 *$ & $-0.055404 * *$ & $0.06661 *$ & $-0.93456 *$ & $-0.000567 *$ & $-0.014007 *$ & $-0.049677 *$ \\
\hline$(0.019885)$ & $(0.021772)$ & $(0.027207)$ & $(0.008907)$ & $(0.006186)$ & $(0.0000574)$ & $(0.002989)$ & $(0.003551)$ \\
\hline
\end{tabular}

Referencias: Errores estándar entre ( ). ***,**,* indican significancia al 90\%, 95\% y 99\% respectivamente. FA: factor de asimetría.

\section{Cuadro 4 \\ Modelos logit para estimar la función de reacción para los distintos tipos de intervenciones}

\begin{tabular}{|c|c|c|}
\hline Constante & $\mathbf{S}_{\mathbf{t}-1}$ & $\boldsymbol{\delta}_{\mathbf{t}-1}$ \\
\hline$-6.8^{*}$ & $1.55^{*}$ & $19.22^{*}$ \\
\hline$(0.213)$ & $(2.361)$ & $(0.427)$ \\
\hline
\end{tabular}

Referencias: entre ( ) se encuentran los desvíos estándar de los estimadores. ***,**, * Indican significancia al $90 \%, 95 \%$ y $99 \%$, respectivamente. 


\section{Cuadro 5}

El efecto medio de las intervenciones ventas mayores a 200 md sobre el TCN

\begin{tabular}{ccccccc}
\hline Variable & Método & $\mathbf{I}$ & SI & Diferencia & $\begin{array}{c}\text { Desv. } \\
\text { Estándar }\end{array}$ & Esta. $\mathbf{T}$ \\
\hline $\mathbf{s}_{\mathbf{t}}$ & SA & 1.86 & -0.01 & 1.86 & 0.09 & 20.5 \\
& EMTT & 1.86 & 2.23 & -0.38 & 0.63 & -0.6 \\
\hdashline $\mathbf{s}_{\mathbf{t}+\mathbf{1}}$ & SA & 0.10 & 0.01 & 0.09 & 0.10 & 0.96 \\
& EMTT & 0.10 & 2.02 & -1.92 & 0.72 & $-2.66^{*}$ \\
\hdashline $\mathbf{s}_{\mathbf{t}+2}$ & SA & -0.52 & 0.02 & -0.54 & 0.10 & -5.62 \\
\hdashline & EMTT & -0.52 & 0.96 & -1.47 & 0.79 & $-1.86 *$ \\
\hdashline $\mathbf{s}_{\mathbf{t}+3}$ & SA & 0.06 & 0.01 & 0.05 & 0.10 & 0.53 \\
\hdashline & EMTT & 0.06 & -0.29 & 0.35 & 0.68 & 0.51 \\
\hdashline $\mathbf{s}_{\mathrm{t}+4}$ & SA & 0.50 & 0.01 & 0.49 & 0.10 & 5.13 \\
\hdashline & EMTT & 0.50 & -0.13 & 0.63 & 0.75 & 0.84 \\
\hdashline $\mathbf{s}_{\mathbf{t}+5}$ & SA & 0.24 & 0.01 & 0.23 & 0.10 & 2.41 \\
& EMTT & 0.24 & -1.33 & 1.57 & 0.54 & $2.92^{*}$ \\
\hline
\end{tabular}

Referencias: I: grupo de observaciones de días en los cuales el BM intervino en el mercado de divisas. SI: grupo de observaciones de días sin intervenciones del BM. SA: Sin agrupar. EMTT: efecto medio del tratamiento sobre las observaciones tratadas. Desv. Estándar: Desvío estándar. Esta. t: estadístico t del EMTT. ***,**, * indican significanacia al $90 \%, 95 \%$ y $99 \%$, respectivamente.

\section{Cuadro 6}

Resultado de la estimación del vector de cointegración entre las variables CIN y AIN (normalizado para CIN)

\begin{tabular}{|c|c|c|}
\hline Constante & AIN $_{\mathbf{t}-\mathbf{1}}$ & $\begin{array}{c}\text { Coeficiente de cointegración } \\
\text { D(CIN) }\end{array}$ \\
\hline-12991.31 & 0.725000 & $-0.180585^{*}$ \\
\hline$(13988.1)$ & $(0.03477)$ & $(0.04994)$ \\
\hline
\end{tabular}

Referencias: Errores estándar entre ( ). ***,**,* indican significancia al 90\%, $95 \%$ y $99 \%$ respectivamente. 
Gráfica 1

Intervenciones del BM en el mercado

de divisas y evolución de la volatilidad del tipo de cambio

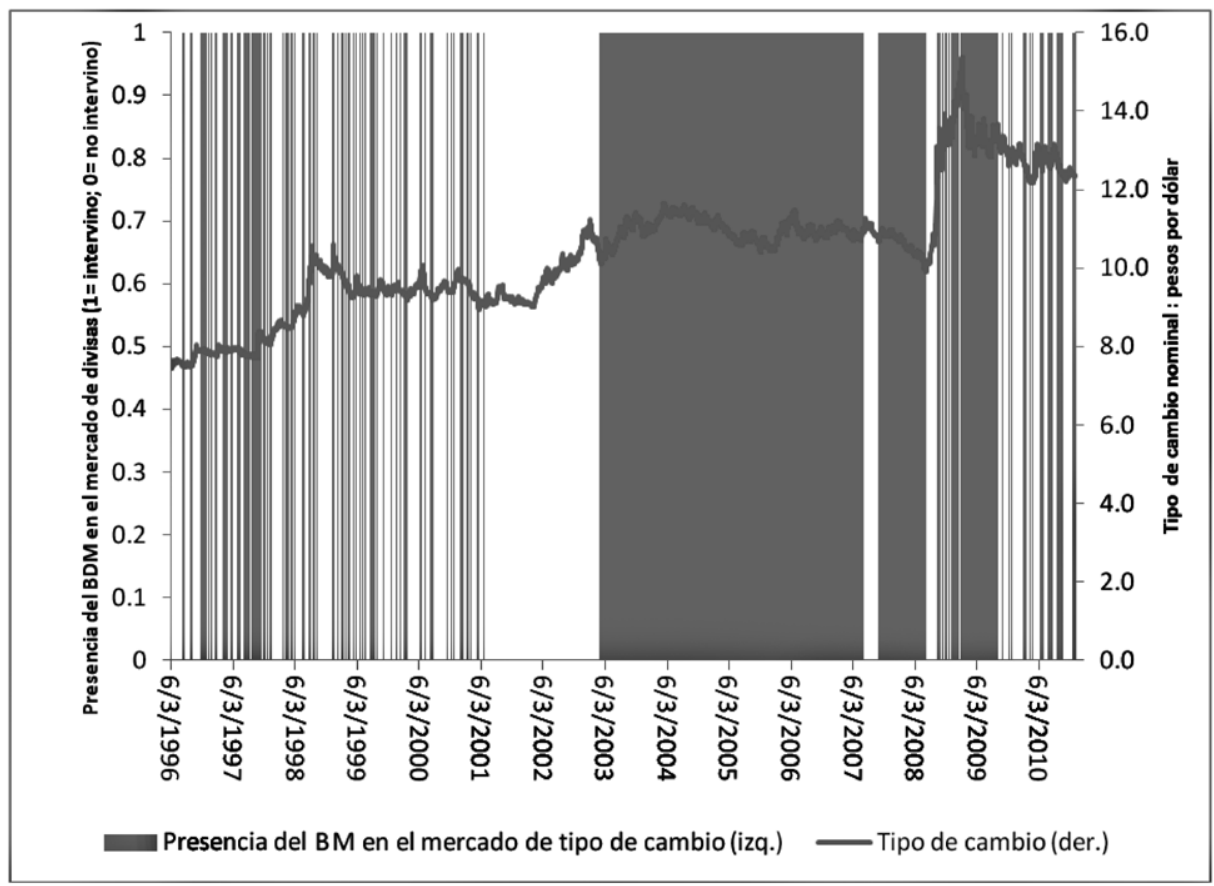

Fuente: Elaboración propia con datos de BM. 


\section{Gráfica 2}

Relación entre la probabilidad de que el BM intervenga (o no) en el mercado de divisas y la variación del TCN

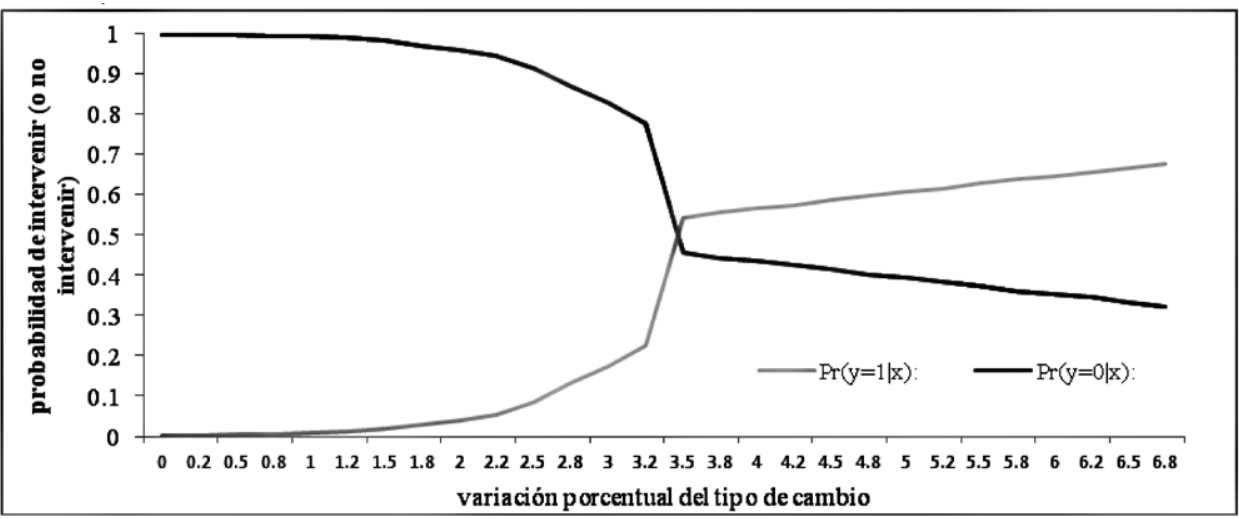

Referencias: $\operatorname{Pr}(\mathrm{y}=1 \mathrm{~lx})$ significa la probabilidad de que el BM intervenga dado el valor de la variación del tipo de cambio en el día anterior. $\operatorname{Pr}(\mathrm{y}=0 \mid \mathrm{x})$ es el evento contrario. En ambos casos la desviación del tipo de cambio respecto a su media se mantiene en su valor promedio histórico.

Gráfica 3

\section{Evolución de la base monetaria, los AIN y el CIN}

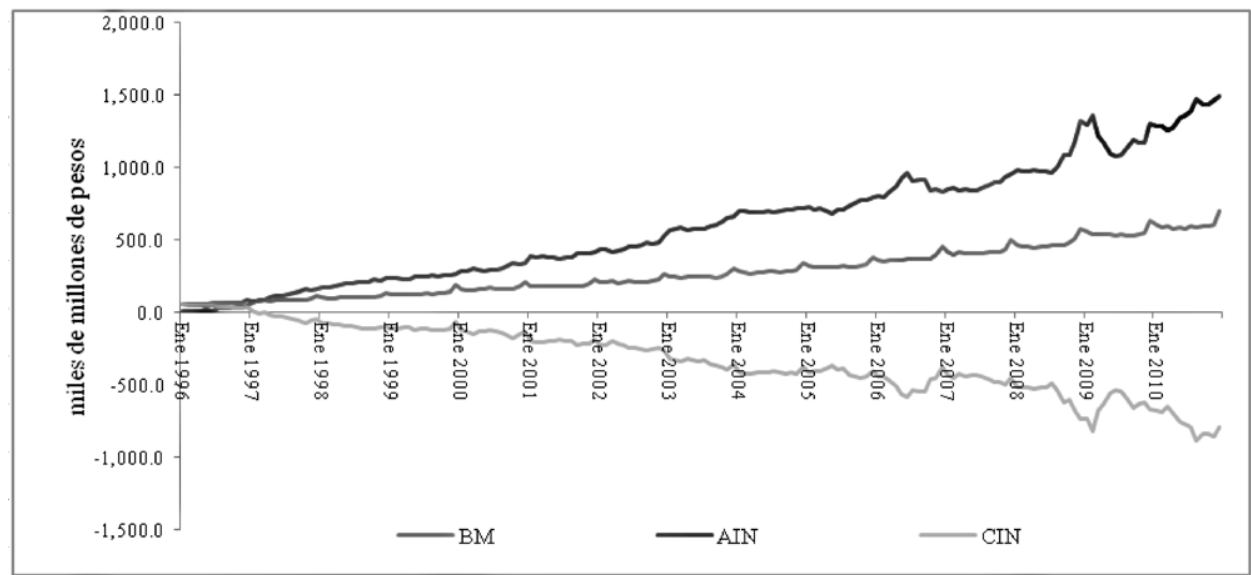

Fuente: Elaboración propia con base en datos del BM. 


\section{Referencias}

Adler, G. y C. Tovar (2011). Foreign Exchange Market Intervention: How Good a Defense Against Appreciation Winds? Regional economic outlook, Western Hemisphere, Washington D.C.

Archer, D. (2005). Foreign exchange market intervention: methods and tactics. BIS Papers (24): 40-55.

Banco de México (1996-2011). Informe Anual: 1995-2010, México D.F.

Bank of International Settlement (BIS), (1997). 26 $6^{\text {th }}$ Conference of Economists, Hobart.

Becker, S. y A. Ichino (2002). Estimation of average treatment effects based on propensity scores. The Stata Journal 2 (4): 358-377.

Benavidez, G. y C. Capistrán (2009). A Note on the Volatilities of the Interest Rate and the Exchange Rate Under Different Monetary Policy Instruments: Mexico 1998-2008. Banco de México, 2009-10.

Bofinger, P. y T. Wollmershäuser (2001). Managed Floating: Understanding the New International Monetary Order. CEPR Discussion Paper 3064.

Bonser-Neal, C. y G. Tanner (1996). Central bank intervention and the volatility of foreign exchange rates: evidence from the options market. Journal of International Money and Finance 15 (6): 853-878.

Caliendo, M. (2005). Some Practical Guidance for the implementation of Propensity Score Matching, IZA Discussion Paper 1588.

Calvo G.A., y C.M. Reinhart (2002). Fear of Floating. Quarterly Journal of Economics 117 (2): 379-408.

Cheung, Y. y M. Chinn (2001). Currency Traders and Exchange Rate Dynamics: A Survey of the U.S. Market. Journal of International Money and Finance 20 (4): 439-471. 
Comisión de Cambios (2010). Comunicado de Prensa del 22 de febrero de 2010.

Domaç, I., y A. Mendoza (2004). Is there Room for Forex Interventions under Inflation Targeting Framework? Evidence from México and Turkey. Policy Research Working Paper 3288, The World Bank.

Domínguez, F. y J. Frankel (1993). Does Foreign Exchange Intervention Matter? The Portfolio Effect. American Economic Review 83 (5): 1356-1369.

y F. Panthaki (2007). The Influence of Actual and Unrequited Interventions. International Journal of Finance and Economics (12): 171-200.

Edison, H. (1993). The effectiveness of Central Bank Intervention: A survey of the Literature after 1982. Special Papers Int. Econ. 18, Princeton University 18.

Enders, W. (2004). Applied Econometric Time Series. 2nd ed., New York: John Wiley and Sons.

Fatum, R. (2010). Foreign Exchange Intervention When Interest Rates Are Zero: Does the Portfolio Balance Channel Matter After All? EPRU WP.

y M. Hutchinson (2006). Evaluating Foreign Exchange Market Intervention: Self-Selection, Counterfactual and Average Treatment Effects. EPRU WP.

Frenkel, M., C. Pierdzioch y G. Stadtmann (2003). The Effects of Japanese Foreign Exchange Market Interventions on the Yen/U.S. dollar Exchange Rate Volatility. Kiel WP 1165.

Galindo, L., y J. Ros (2008). Alternatives to inflation targeting in Mexico. International Review of Applied Economics 22 (2): 201-214.

Gandolfo, G. (2000). International Economics. Berlin: Springer-verlag.

Glick, R., X. Guo y M. Hutchison (2004). Currency Crises, Capital Account Liberalization, and Selection Bias. Federal Reserve Bank Of San Francisco, WPS 15. 
Guimarães, R. y C. Karacadag (2004). The Empirics of Foreign Exchange Intervention in Emerging Market Countries: The Cases of México and Turkey. IMF WP 123.

Harvey, J. (2008). Currencies, Capital Flows, and Crises: A Post Keynesian Analysis of Exchange Rate Determination. London: Routledge.

Hüfner, F.(2004).Foreign Exchange Intervention as a Monetary Policy Instrument. Mannhein: Center for European Economic Research.

Humpage, O. y J. Ragnartz (2005). Swedish Intervention and the Krona Float, 1993-2002. Federal Reserve of Cleveland, WP Diciembre.

Hung, J. (1997). Intervention Strategies and Exchange Rate Volatility: A Noise Trading Perspective. Journal of International Money and Finance 16 (5): 779-773.

Imbens, G. (2004). Nonparametric estimation of average treatment effects under exogeneity: a review. The Review of Economics and Statistics 86 (1): 4-29.

Ito, T. y T. Yabu (2004). What promotes Japan to intervene in the forex market?: A new approach to a reaction functions. NBER WP 10456.

Kim, S. (2003). Monetary Policy, Foreign Exchange intervention, and the Exchange Rate in a Unifying Framework. Journal of International Economics 60 (2): 355-86.

Leuven, L. y B. Sianesi (2003). PSMATCH2: Stata module to perform full Mahalanobis and propensity score matching, common support graphing, and covariate imbalance testing. Statistical Software Components S432001, Boston College Department of Economics.

Lin, S. y H. Ye (2007). Does inflation targeting really make a difference? Evaluating the treatment effect of inflation targeting in seven industrial countries. Journal of Monetary Economics 54 (8): 2521-2533.

Lyons, R.(2001). The Microstructure Approach to Exchange rate. Cambridge. 
Mántey, G. (2009). Intervención esterilizada en el mercado de cambios en un régimen de metras de inflación: la experiencia de México. Investigación Económica LXVIII [63]: 47-78.

Neely, C. (2005). An Analysis of Recent Studies of the Effect of Foreign Exchange Intervention. Federal Reserve Bank of St. Louis Review Noviembre-Diciembre.

Perrotini, I. (2012). Neo-Wicksellian Economics and the New Monetary Consensus in Small Open Economies: Theory and Empirical Evidence. North Carolina: Durham Basic Books.

Rosenbaum, P., y D. Rubin (1983). The Central Role of the Propensity Score in Observational Studies for Causal Effects. Biometrika (70): 41-55.

Sarno, L. y M. P. Taylor. (2002). The Economics of Exchange Rates. Cambridge: Cambridge University Press.

Sarno, L., y M. Taylor (2001). Official Intervention in the Foreign Exchange Market: Is It Effective and, if so, How Does it Work?. Journal of Economic Literature XXIX [39]: 839-868.

Svensson, L. (2001). Independent Review of the Operation of Monetary Policy in New Zealand: Report to the Minister of Finance. Febrero.

Svensson, L. (2010). Inflation Targeting. Friedman, Benjamin M., y Michael Woodford, eds. Handbook of Monetary Economics Vol. 3a y 3b forthcoming.

Taylor, J. B. (1999). An Historical Analysis of Monetary Policy Rules. John B. Taylor, ed. Monetary Policy Rules. Chicago: University of Chicago Press.

Vitale, P.(2006). A market microstructure analysis of foreign exchange intervention. ECB, WPS 629.

Wilde, O. (1894). Phrases and Philosophies for the Use of the Young. En The Complete Stories, Plays and Poems. Londres:Tiger Books International PLC. 
Woodford, M. (2003). Interest and Prices: Foundations of a theory of Monetary Policy. Princeton, New Jersey: Princeton University Press.

Wooldridge, J. M. (2004). Econometric Analysis of Cross Section and Panel Data. London The Mit Press. (A) 